\title{
Survey of difficult to contain and treat children and adolescents
}

\author{
Jan Sebestik, M Elena Garralda
}

\begin{abstract}
Child psychiatrists and paediatricians are faced by a number of disturbed children and adolescents who become a major treatment challenge due to a combination of their dangerous and/or disruptive behaviour and the unavailability of resources. Paediatricians and psychiatrists in one regional health area were questioned about the number of such children and adolescents under 18 years of age seen over a 20 month period. The response rate was $44 \%$ (86/194 specialists approached). A total of 72 children and adolescents were identified. For a health district with a total population of 200000 , this represents nearly five children (about one of these identified by paediatricians and one with associated learning disability). The majority of cases were adolescents with chronic problems (over one month's duration) but most cases reported by paediatricians were acute in preadolescents. Conduct disorders, autistic spectrum, and psychotic disorders were the most common clinical problems.

Seriously disruptive behaviour had been present in two thirds and marked problems with behaviour that was a physical risk to others and to the self were present in over half and nearly a third of cases respectively. Services had been universally stretched by these children's difficulties. Clinicians thought the needs of these children would have been best met either through psychiatric day hospital type facilities or in combined paediatricl child psychiatric inpatient units. (Arch Dis Child 1996;75:78-81)
\end{abstract}

Keywords: child psychiatric disorders, disruptiveness, dangerousness, service provision.

Child psychiatrists and paediatricians are faced by a number of disturbed children and adolescents who become a major challenge due to a combination of their dangerous and/or disruptive behaviour and the unavailability of resources. Although previous surveys suggest that any one health district is unlikely to encounter more than one or two cases each year, ${ }^{1}$ these children and adolescents can cause considerable anxiety for professionals and disruption of services, as well as the difficulty in providing satisfactory treatment for them.

The unavailability of adequate service provision for these children and adolescents may become apparent when there is a request for hospital admission from community or outpatient services, ${ }^{2}$ but often difficulties in management become apparent only after hospital admission, for example to paediatric, medical, or adult psychiatric wards. The variable and incomplete adolescent psychiatric services have been highlighted for adolescents who require inpatient care. ${ }^{3}$ This may lead to unsatisfactory and even legally contested admissions to adult wards. ${ }^{4}$ Sometimes the unavailability of resources is time related, such as perceived delay in response to acute situations and the criticism of inadequate weekend provision from regional child and adolescent psychiatric units.

Solutions to the problem of managing disturbed children and adolescents have been considered. For example, there has been tentative evidence that children and adolescents with a range of psychiatric disorders can be managed on paediatric wards with staff without psychiatric qualifications, providing there is support from local child psychiatric services. ${ }^{5}$ However this is unlikely to be sufficient to contain severely disruptive and dangerous behaviour in children.

This project aimed to establish the nature and extent of the problem, to describe the clinical picture, to define the nature of the disruptive/dangerous behaviours, and to identify possibly helpful management strategies.

\section{Method}

A questionnaire was sent to all paediatricians, child and adolescent psychiatrists, learning disability psychiatrists, and general psychiatrists within the North West Thames Region, requesting them to identify children and adolescents under 18 years of age seen over a 20 month period between January 1991 and August 1992 where the unavailability of resources to adequately contain their behaviour had made psychiatric/paediatric assessment and treatment difficulty to institute or maintain.

Cases identified by child psychiatrists and paediatricians were followed up with a telephone or personal semistructured interview with the clinician to gain information on the medical and psychiatric diagnoses and the

\author{
Jlace, Lo Sebestik \\ ME Garralda \\ Correspondence to: \\ Professor Garralda. \\ Medical School, \\ Academic Unit of \\ Child and Adolescent
}


Table 1 Questionnaire study: cases identified by specialty

\begin{tabular}{|c|c|c|c|c|c|}
\hline & Paediatricians & $\begin{array}{l}\text { Child/adolescent } \\
\text { psychiatrists }\end{array}$ & $\begin{array}{l}\text { Learning disabilities } \\
\text { consultants }\end{array}$ & Adult psychiatrists & Total \\
\hline Response rate & $29 / 73(40 \%)$ & $26 / 35(74 \%)$ & $10 / 17(59 \%)$ & $21 / 69(30 \%)$ & $86 / 194(44 \%)$ \\
\hline No of cases & $12 / 13 \star$ & 31 & 12 & 17 & $72 / 73$ * \\
\hline Mean No of cases/clinician & 0.4 & 1.1 & 1.2 & 0.8 & 0.8 \\
\hline \multicolumn{6}{|l|}{ Age / (years) } \\
\hline$<12$ & 8 & 3 & 2 & 0 & 13 \\
\hline $12-15$ & 3 & 17 & 5 & 2 & 27 \\
\hline $16-18$ & 1 & 11 & 5 & 15 & 32 \\
\hline Problems/conditions $\dagger$ & $(n=24)$ & $(n=39)$ & $(n=29)$ & $(n=26)$ & $(n=118)$ \\
\hline Conduct disorder/behavioural problems & 6 & 20 & 11 & 9 & 46 \\
\hline Developmental/autistic spectrum & 7 & 1 & 17 & 1 & 26 \\
\hline Psychoses & 1 & 6 & 0 & 9 & 16 \\
\hline Affective/personality disorders & 2 & 6 & 0 & 4 & 12 \\
\hline Other psychiatric disorders & 3 & 4 & 1 & 2 & 10 \\
\hline
\end{tabular}

* One case identified by two paediatricians.

† Not mutually exclusive.

nature and severity of the dangerous/disruptive behaviours. Questions about service provision and the professionals involved as well as basic demographic data were also included.

\section{Results and comment}

QUESTIONNAIRE STUDY

Results

One hundred and ninety four consultants were identified. The paediatric group of 73 included 50 hospital paediatricians, six community paediatricians, and 17 senior community medical officers. Details on response rates, on age, and problem/diagnoses are given in table 1 .

The total response to the questionnaires was 44\%, with highest rates for child psychiatrists and lowest for adult psychiatrists. Thirty per cent of consultants completing the questionnaires $(26 / 86)$ reported cases, with the highest rate of reporting among consultants in learning disability $(60 \%$ or $10 / 17)$. Seventy three cases ( 72 as one child was identified by two responders) were identified with mean responder rates of 0.8 .

The age range for cases reported was 3 to 18 years. The majority $(82 \%)$ were adolescents over 12 years of age but there were differences according to specialty. Most children reported by paediatricians were preadolescents and the majority of cases reported by adult psychiatrists were between 16 and 18 years of age. The sex distribution for all reported cases indicated a slight predominance of males $(58 \%)$ over females (42\%), a pattern that was consistent across specialties.

One hundred and eighteen main problems or diagnoses were given for 68 cases. Most common were conduct disorders/behaviour problems, followed by developmental delays and autistic spectrum disorders, schizophrenic and other psychotic states, affective and personality disorders. Most developmental problems were reported by paediatricians and consultants in learning disability and most psychotic states by other psychiatrists. Medical problems were reported in nine, mostly by paediatricians. Medical problems included epilepsy, hemiplegia, Angelman's syndrome, and brain injury.

\section{Comment}

Our results identify a perceived gap in the provision of health services for children and adolescents. For a total regional population of
2981079 consultant paediatricians and psychiatrists identified 72 cases where the unavailability of resources to adequately contain behaviour had made psychiatric/paediatric assessment and treatment difficult to institute or maintain over the previous 20 months. This represents close to five (4.8) children and adolescents for a health district with a total general population of 200000 , with about one out of these five having developmental/learning problems, and one being a main concern to paediatricians.

These figures are based on a low response rate and subject to retrospective bias and they are most likely underestimates. However it seems probable that clinicians more actively involved with these sort of cases would be more likely to remember and feel motivated to reply. When we checked with non-responders it was clear that some hospital paediatricians had not seen any such cases and had not replied because of this. Our figures in fact are close to those derived from other comparable though not identical surveys, for example those reported by Meadow from a national survey of paediatricians, ${ }^{1}$ and the combined figures from unpublished reports on regional surveys on children and adolescents with challenging behaviours and learning/developmental problems (M E Garralda, 1991; J Cass et al, 1994; A Gath, 1991).

Most children and adolescents with problems similar to those identified here may be expected to be treated in psychiatric inpatient units for children or adolescents. In the region surveyed at the time of the study there were three such units: an eight bedded unit for children, a 20 bedded adolescent unit for 12-16 year olds, and a 20 bedded unit for adolescents over 16 years of age. This would represent five beds for a total district population of 200000 (approximately one for preadolescents, 1.5 for young and two for older adolescents). Our figures would indicate that this was insufficient. However before concluding that more inpatient beds are required it seems important to consider in more detail the nature of the challenge presented by these children and suggested solutions by clinicians. This is available from our interview study.

In doing this it must be pointed that our survey is confined to children and adolescents who are a major assessment and treatment problem to clinicians in health settings. There 
Table 2 Interview information

\begin{tabular}{|c|c|c|c|}
\hline & $\begin{array}{l}\text { Paediatric cases } \\
(n=11)\end{array}$ & $\begin{array}{l}\text { Child/adolescent } \\
\text { psychiatric cases } \\
(n=15)\end{array}$ & Total $(n=26)$ \\
\hline \multicolumn{4}{|l|}{ Age (years) } \\
\hline$<12$ & 7 & 2 & 9 \\
\hline$>12$ & 4 & 13 & 17 \\
\hline \multicolumn{4}{|l|}{ Length of problem } \\
\hline Acute $(<1 / 12)$ & 4 & 1 & 5 \\
\hline Chronic ( > 1/12) & 7 & 4 & 11 \\
\hline Learning disability & 6 & 2 & 8 \\
\hline Medical problem & 9 & 0 & 9 \\
\hline \multicolumn{4}{|l|}{ Social problems } \\
\hline Family breakdown & 5 & 11 & 16 \\
\hline Other adverse factors & 2 & 13 & 15 \\
\hline \multicolumn{4}{|c|}{ No of placements excluding home } \\
\hline $0-1$ & 2 & 12 & 14 \\
\hline $2-4$ & 9 & 3 & 12 \\
\hline \multicolumn{4}{|c|}{ No of professionals involved } \\
\hline $1-2$ & 4 & 5 & 9 \\
\hline $3-4$ & 7 & 8 & 15 \\
\hline $5+$ & 0 & 2 & 2 \\
\hline
\end{tabular}

are an important additional number of adolescents with psychiatric problems known to social services and penal institutions that have been the subject of other surveys and are not included here (P Garfield et al, 1995).

THE INTERVIEW STUDY

In order to gain a more detailed account of the subjects' difficulties the questionnaires were followed up with a semistructured interview with clinicians. We confined this part of the survey to cases reported by child specialists. It proved possible to arrange interviews for 26 cases: all but one of those reported by paediatrician (11 cases) and half those reported by child and adolescent psychiatrists (15). The age and sex distribution of this subsample were in line with those in the questionnaire study with an age range of 3-18 years and a slight excess of males over females (14 and 12 respectively). Under 12 year olds and boys were more common among cases referred by paediatricians whereas adolescents and girls were more often reported by child and adolescent psychiatrists.

\section{Results}

Twenty eight psychiatric diagnoses were given (according to the World Health Organisation classification).$^{6}$ In line with the questionnaire survey, conduct disorders were most prominent in both groups (15 subjects). Autistic spectrum diagnoses were present in four, schizophrenia/mania in four, eating disorders in three, and adjustment reactions in two. Associated learning disability and medical problems were present in nearly a third of cases and were mostly reported by paediatricians. Medical problems included hemiplegia, epilepsy, respiratory infection, and Angelman's syndrome.

We examined the nature of the challenging behaviour that had impeded the children's medico/psychiatric assessment and treatment by defining dangerous and disruptive behaviour as follows: risk of harm to others, risk of self harm, and disruption to routine. Each variable was rated on a six point scale.

Low harm scores ( $0-2)$ indicated concern about possible harm. 'Moderate' harm (score 3) to others included hitting, pinching, and kicking without causing injury. 'Severe' harm (scores 4-5) included these behaviours, but severe enough to cause injury such as bruising, fractured arm and jaw in one case, 'broken' finger in another case, attacking with objects, and also one case of repeated arson.

'Moderate' self harm included scratching, head banging, hair pulling, and accidental self injury as a result of aggressive or disruptive behaviour. 'Severe' self harm included these behaviours, but to a greater degree, causing injury that required attention and, in addition, deliberate self harm through drug overdose or wrist cutting.

Disruptive behaviour was measured by effect on routine, rather than particular types of behaviour. Examples included shouting, damage to property, restlessness, running away, distracting others, throwing food, slamming doors, and swearing. In addition threatening or aggressive behaviour that is disruptive by the attention attracted was included.

Figure 1 details rates of problems in each of these three areas for the whole group. It can be seen that severely disruptive behaviour was prominent in the majority of cases and that this was more often a problem than risk to self or others. However a substantial number of children (15) were showing moderate or severe behaviours that were a physical risk to others and eight were exhibiting self injurious behaviours. The pattern of behaviours was comparable for the cases reported by paediatricians and child psychiatrists.

In a minority of five cases, mostly reported by paediatricians, medical investigations were the principal component of the management plan impeded by the challenging behaviour. For the majority, however, management included treatment plans for psychological disorder involving the use of medication, behaviour/ cognitive therapies, family therapy, psychodynamic therapy, and other treatment such as group therapy, inpatient and day patient programmes, and special educational placements.

Details on the duration of the problem, associated psychosocial disruption, placements, and professionals involved are given in table 2 .

The management challenge presented by these children and adolescents could be subdivided into acute (a fifth of cases) where the difficulty in containment, investigation, and treatment had lasted up to one month and chronic cases (the majority) where the difficulties lasted from one month to one year or longer. In

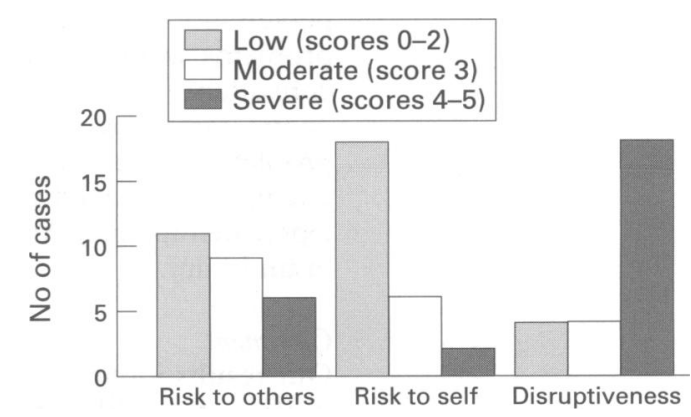

Figure 1 Disruptive and dangerous behaviour. 
fact in nearly half the cases the problems had lasted more than one year. Acute problems were more common among cases reported by paediatricians.

The various settings where it had proved difficult to contain the child were the parents' or relatives' homes (24 cases), local authority placements such as residential establishments or foster homes (9), paediatric units (4), and psychiatric inpatient or day patient facilities (5). As table 2 shows, in two thirds of cases there had been three or more different professionals from health, social, and educational services involved with the problem and in nearly half there had been two or more placements for the child or adolescent away from home or principal residence.

A variety of associated adverse psychosocial factors were identified relevant to the chronic difficulty. The most common factor was disruption of the family by separations, but other factors reported mainly by child and adolescent psychiatrists were removal from the home, abuse, relationship difficulties with parents, absence and loss of a parent, family history of alcohol abuse, and mental disorder. Family breakdown was common and in only a third of cases were the children living with both natural parents. Interestingly all children with a diagnosis of a psychotic disorder and nearly all those with an autistic disorder were living with their two natural parents and these cases also had the least number of adverse social factors.

The clinical status of 25 cases at the time of the assessment was examined. In the majority (17) there remained significant dangerous/ disruptive behaviour despite some degree of investigation and treatment that had been instituted in all but two cases.

Clinicians were asked what resources or facilities may have provided adequate containment to allow investigations or treatment to take place. Day facilities were most frequently proposed (15 cases) and fell into two main categories: firstly health based psychiatric day hospitals and special educational units with psychiatric input and jointly run by educational and health services. The other preferred facility (seven cases) was a combined paediatric/child psychiatric inpatient unit. Modifications of this idea included a neuropsychiatric unit and a paediatric ward with a designated area with psychiatrist, psychologist, and psychiatric nurse input.

Seventeen different facilities were actually sought within health, social and educational services, within and outside the region. Most frequently mentioned were adolescent psychiatric inpatient units (four cases) and boarding schools and social service residential units (three instances of each). The perceived availability of these resources by clinicians was rated. The most common problems encountered were facilities being available but not for long enough or being available after an excessive time delay.

\section{Comment}

This detailed analysis of cases reported by paediatricians and child psychiatrists confirmed that the disruption and physical threat to others and themselves caused by this small group of children and adolescents was considerable and presented a major challenge to clinicians.

Five acute cases were identified and if our figures are not gross underestimates this would mean than a standard health district with a population of 200000 would see fewer than one such child every three years. While potentially very disruptive this is therefore a rare occurrence. It may require individually based strategies at the acute unit, probably involving flexibility to employ additional child and adolescent psychiatric nurses or nurses with comparable expertise on a short term basis.

The majority of cases were chronic. They appeared very costly in terms of resources used and disruption caused and they had proved very intractable. In some cases dysfunctional social circumstances may have compounded conduct and other disorders in the children and adolescents, but in a proportion it seemed that intrinsically severe problems causing disorganisation to the individual's personality functioning and mental integration, such as autism and schizophrenic illness, were sufficient in themselves to cause major disruptive behaviour. The high representation of learning disabilities among the sample highlights the importance that any management strategies proposed take account of the needs of this group.

It seems unlikely that more psychiatric inpatient facilities for children and adolescents would have solved the clinical problem as this was hardly mentioned as a solution by clinicians and some of the youngsters were in fact not adequately contained in psychiatric units. What seems to be required is the availability of regional psychiatric day units with educational facilities or jointly run educational health provision that are able to attend to some 25-50 adolescent referrals of under 18 year olds over one to two years, and able to work on a comparatively long term basis in order to devise and implement optimal management strategies. The small numbers of children under 12 (eight were identified in our survey) makes specific unit based proposals impracticable for this group. A better alternative may be flexibility in the age range treated by this type of unit in order to accommodate this group. Units of this kind could well be very cost effective given the high use of resources made by the children identified in our survey.

1 Meadow R Dangerous, disruptive children. London: British Paediatric Association, 1990.

2 Sutton A, Modell M, Kaplan C, Russell J, Burrows J. Legal and ethical issues in child psychiatry. Psychiatric Bulletin 1989; 13: 193-6.

3 Health Advisory Service. Bridge over troubled waters. London: HMSO, 1986.

4 Dyer C. Placing children in adult psychiatric wards. $B M Y$ 1992; 304: 462-3.

5 Kolind A, Maratos J. Can children with psychiatric disorder be treated in a general ward? $B M F$ 1982; 91: 257-8.

6 World Health Organisation. The ICD-10 classification of mental and behavioural disorders. Geneva: WHO, 1994. 